\title{
Beam shaping for holographic techniques
}

\section{Alexander Laskin, Vadim Laskin, Aleksei Ostrun}

Alexander Laskin, Vadim Laskin, Aleksei Ostrun, "Beam shaping for holographic techniques," Proc. SPIE 9200, Photonic Fiber and Crystal Devices: Advances in Materials and Innovations in Device Applications VIII, 92000E (5 September 2014); doi: 10.1117/12.2061222

SPIE Event: SPIE Optical Engineering + Applications, 2014, San Diego, California, United States 


\title{
Beam shaping for holographic techniques
}

\author{
Alexander Laskin ${ }^{\mathrm{a}}$, Vadim Laskin ${ }^{\mathrm{a}}$, Aleksei Ostrun ${ }^{\mathrm{b}}$ \\ ${ }^{a}$ AdlOptica GmbH, Rudower Chaussee 29, 12489 Berlin, Germany \\ ${ }^{b}$ St. Petersburg National Research University of Information Technologies, \\ Mechanics and Optics, Kronverkskiy pr, 49, 197101, St.Petersburg, Russia,
}

\begin{abstract}
Uniform intensity of laser radiation is very important in holographic and interferometry technologies, therefore transformation of typical Gaussian distribution of a $\mathrm{TEM}_{00}$ laser to flat-top (top hat) is an actual technical task, it is solved by applying beam shaping optics. Holography and interferometry have specific requirements to a uniform laser beam, most important of them are flatness of phase front and extended depth of field. There are different refractive and diffractive beam shaping approaches used in laser industrial and scientific applications, but only few of them are capable to fulfil the optimum conditions for beam quality demanding holography and interferometry. We suggest applying refractive field mapping beam shapers piShaper, which operational principle presumes almost lossless transformation of Gaussian to flat-top beam with flatness of output wavefront, conserving of beam consistency, providing collimated low divergent output beam, high transmittance, extended depth of field, negligible wave aberration, and achromatic design provides capability to work with several lasers with different wavelengths simultaneously. This approach is used in SLM-based technologies of Computer Generated Holography, Dot-Matrix mastering of security holograms, holographic data storage, holographic projection, lithography, interferometric recording of Volume Bragg Gratings. High optical quality of resulting flat-top beam allows applying additional optical components to vary beam size and shape, thus adapting an optical system to requirements of a particular application. This paper will describe design basics of refractive beam shapers and optical layouts of their applying in holographic systems. Examples of real implementations and experimental results will be presented as well.
\end{abstract}

Keywords: beam shaping, flat-top, top-hat, holography, interferometry, volume Bragg grating, Holographic Data Storage, Computer Generated Holography, homogenizing.

\section{INTRODUCTION}

Various implementations of holographic and interferometric techniques, for example Computer Generated Holography (CGH), Holographic data Storage (HDS), Dot-Matrix hologram mastering or multi-colour Denisyuk holography use the Spatial Light Modulators (SLM) like Liquid Crystal on Silicon (LCoS) or Digital Mirror Devices (DMD) as versatile tools to control the laser radiation. Typically the intensity distribution of laser sources is described by Gaussian function provided by physics of creating the laser radiation. This Gaussian profile is naturally inhomogeneous, and in SLM-based applications this inhomogeneity of intensity is rather a source of problems: variation of brightness of reproduced images, instability of recording processes, reduced image contrast, inconvenience in realization of optical setups. Therefore, the task of transformation of laser beam intensity profile to a uniform one (flat-top, tophat) is an actual task for SLM-based applications. A specific demand of SLM illumination in these techniques is in strict requirements to flatness of phase front of a laser beam that should be conserved while any irradiance profile transformations, i.e. flatness of both phase front and irradiance distribution should be realized simultaneously. There are several beam shaping techniques applied in modern laser technologies, some of them, like integration systems based on arrays of microlenses, micromirrors, prisms, cannot be applied since their physical principle implies destroying the beam structure and, hence, leads to loss of spatial coherence. Other techniques: truncation of a beam by an aperture, attenuation by apodizing filters allow obtaining acceptable in many cases homogeneity of irradiance profile, but evident disadvantage of these techniques is essential loss 
of costly laser energy. To meet the demands of holography it is suggested to apply beam shaping systems built on the base of field mapping refractive beam shapers like $\pi$ Shaper, which operational principle implies almost lossless transformation of laser irradiance distribution from Gaussian to flattop, conserving of beam consistency, flatness of output phase front, low divergence of collimated output beam, high transmittance, extended depth of field, capability to operate with $\mathrm{TEM}_{00}$ or multimode lasers, implementations as telescopes or collimators. This article describes basic principles and important features of refractive beam shapers as well as some optical layouts that can be built on their base to meet requirements of modern holographic techniques.

\section{FIELD MAPPING REFRACTIVE BEAM SHAPERS}

\subsection{Basics of optical design}

The design principles of refractive beam shapers of field mapping type, like $\pi$ Shaper, are well-known and described in the literature ${ }^{1,5,6,7,8,9}$. Most often these devices are implemented as telescopic systems with two optical components, it is implied that wave fronts at input and output are flat, the transformation of irradiance profile from Gaussian to uniform is realized in a controlled manner, by accurate introducing of wave aberration by the first component and further its compensation by the second one, Fig.1, top. Thus, the resulting collimated output beam has uniform irradiance and flat wave front; it is characterized by low divergence - almost the same like one of the input beam. In other words, the field mappers transform the irradiance distribution without deterioration of the beam consistency and without increasing of beam divergence. Shortly the main features of refractive field mappers are:

- refractive optical systems transforming Gaussian to flat-top (uniform) irradiance distribution;

- transformation through controlled phase front manipulation $-1^{\text {st }}$ component introduces spherical aberration required to re-distribute the energy, then $2^{\text {nd }}$ component compensates the aberration;

- output beam is free of aberrations, phase profile is maintained flat, hence, low output divergence;

- $\mathrm{TEM}_{00}$ and multimode beams applied;

- collimated output beam;

- resulting beam profile is stable over large distance;

- implementations as telescopic or collimating optical systems;

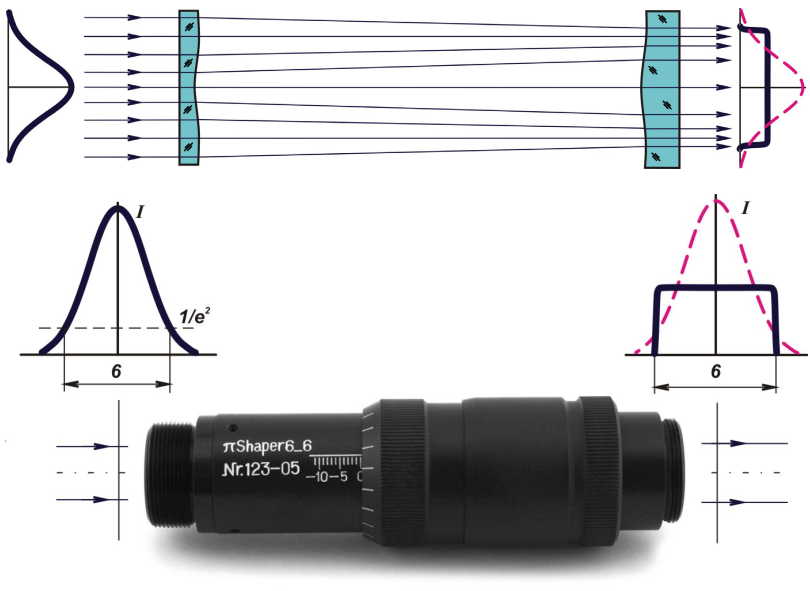

Figure 1 Refractive field mapping beam shaper $\pi$ Shaper
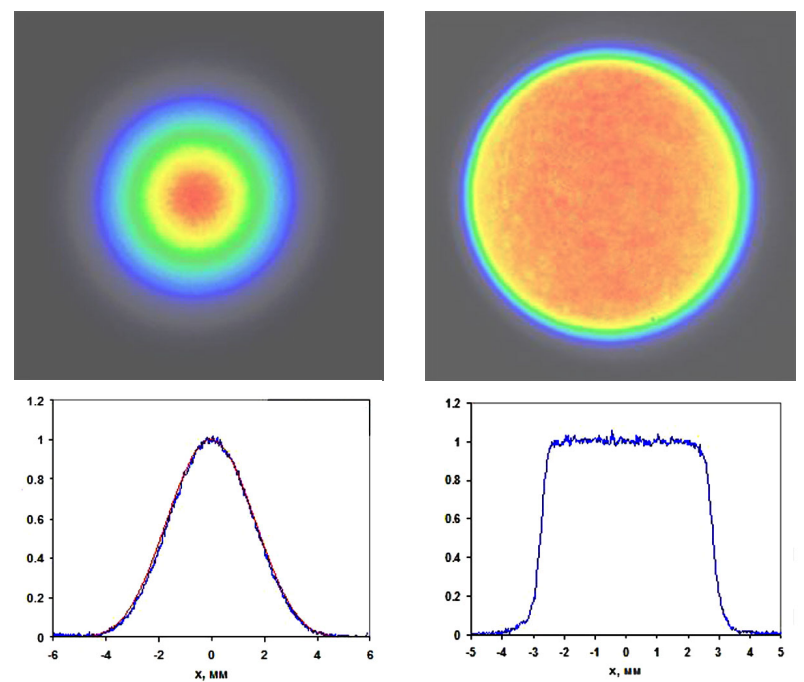

Figure 2. Experimental intensity profiles: Left - Input $\mathrm{TEM}_{00}$ beam, Right - after the $\pi$ Shaper

- achromatic optical design, hence the beam shaping is provided for a certain spectral range simultaneously;

- Galilean design, no internal focusing.

Example of beam shaping for Nd:YAG laser is presented in Fig.2. 


\subsection{Propagation of flattop beams in space}

It is usual to characterize beam shaping optics by the working distance - the distance from last optical component to a plane where a target irradiance profile, flattop or another one, is created. The working distance is an important specification for diffractive beam shapers and refractive homogenizers (or integrators) based on multi lens arrays. But in case of the field mapping beam shapers the output beam is collimated and, hence, instead of a definite plane where a resulting irradiance profile is created, there exists certain space after a beam shaper where the profile is kept stable. In other words, the working distance isn't a specification for the field mapping beam shapers, it is better specify the depth of field (DOF) after a beam shaper where resulting irradiance profile is stable. This DOF is defined by diffraction effects happening while a beam propagating and depends on wavelength and beam size.

When a $\mathrm{TEM}_{00}$ laser beam with Gaussian irradiance distribution propagates in space its size varies due to inherent beam divergence but the irradiance distribution stays stable, this is a famous feature of $\mathrm{TEM}_{00}$ beams that is widely used in practice. But this brilliant feature is valid for Gaussian beams only! When light beams with non-Gaussian irradiance distributions, for example flattop beams, propagate in space, they get simultaneously variation of both size and irradiance profile. Suppose a coherent light beam has uniform irradiance profile and flat wave front, Fig. 3, this is a popular example considered in diffraction theory ${ }^{2,3,4}$, and is also a typical beam created by field mapping refractive beam shapers converting Gaussian to flattop laser beam.

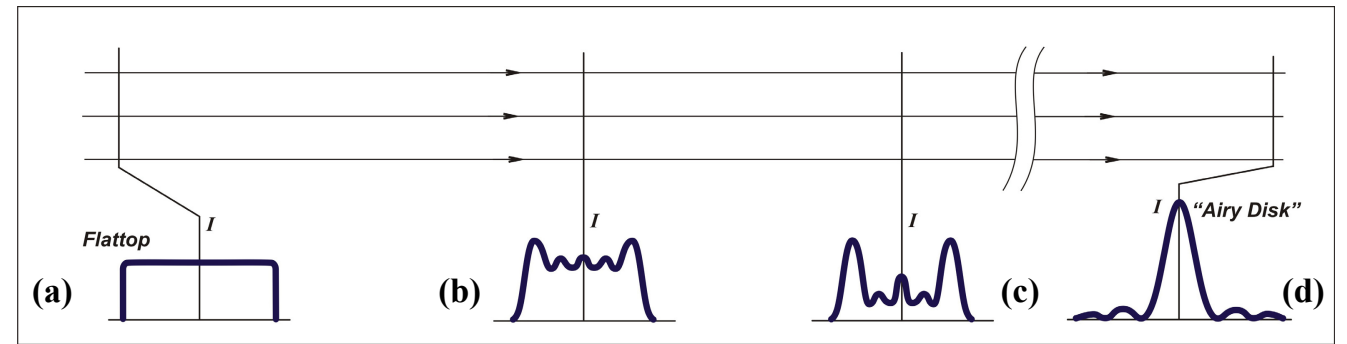

Figure 3 Irradiance profile variation by a flattop beam propagation.

Due to diffraction the beam propagating in space gets variation of irradiance distribution, some typical profiles are shown in Fig. 3: at certain distance from initial plane with uniform irradiance distribution (a) there appears a bright rim (b) that is then transformed to more complicated circular fringe pattern (c), finally in infinity (so called far field) the profile is featured with relatively bright central spot and weak diffraction rings $(d)$ - this is the well-known "Airy disk" distribution described mathematically by formula

$$
I(\rho)=I_{0}\left[J_{1}(2 \pi \rho) /(2 \pi \rho)\right]^{2}
$$

where $I$ is irradiance, $J_{I}$ is the Bessel function of $1^{\text {st }}$ kind, $1^{\text {st }}$ order, $\rho$ is polar radius, $I_{0}$ is a constant.

The "Airy disk" function is result of Fourier-Bessel transform for a circular beam of uniform initial irradiance,

Evidently, even a "pure" theoretical flattop beam is transformed to a beam with essentially non-uniform irradiance profile. There exists, however, certain propagation length where the profile is relatively stable, this length is in reverse proportion to wavelength and in square proportion to beam size. For example, for visible light, single mode initial beam and flattop beam diameter $6 \mathrm{~mm}$ after a $\pi$ Shaper $6 \_6$ the length where deviation from uniformity doesn't exceed $\pm 10 \%$ is about $200-300 \mathrm{~mm}$, for the $12 \mathrm{~mm}$ beam it is about 1 meter.

There are many laser applications where conserving a uniform irradiance profile over certain distance is required, for example holography, interferometry; the extended DOF is also very important in various industrial techniques to provide less tough tolerances on positioning of a work piece. As a solution to the task of providing a necessary resulting spot size with conserving the flattop profile over extended DOF it is fruitful to apply imaging techniques that are considered in next chapter. 


\section{IMAGING OF FLATTOP BEAMS}

\subsection{Telecentric imaging of $\pi$ Shaper output}

Imaging technique is a powerful tool to building complex beam shaping systems on the base of refractive beam shapers like $\pi$ Shaper, essential features of this approach are considered in paper $^{12}$. Here we emphasize on most important for practice aspects and consider in details the telecentric imaging system, Fig. 4, that is practically a perfect tool to magnify or de-magnify the laser beams in holography to illuminate SLM with conserving the flatness of phase front and irradiance profile.

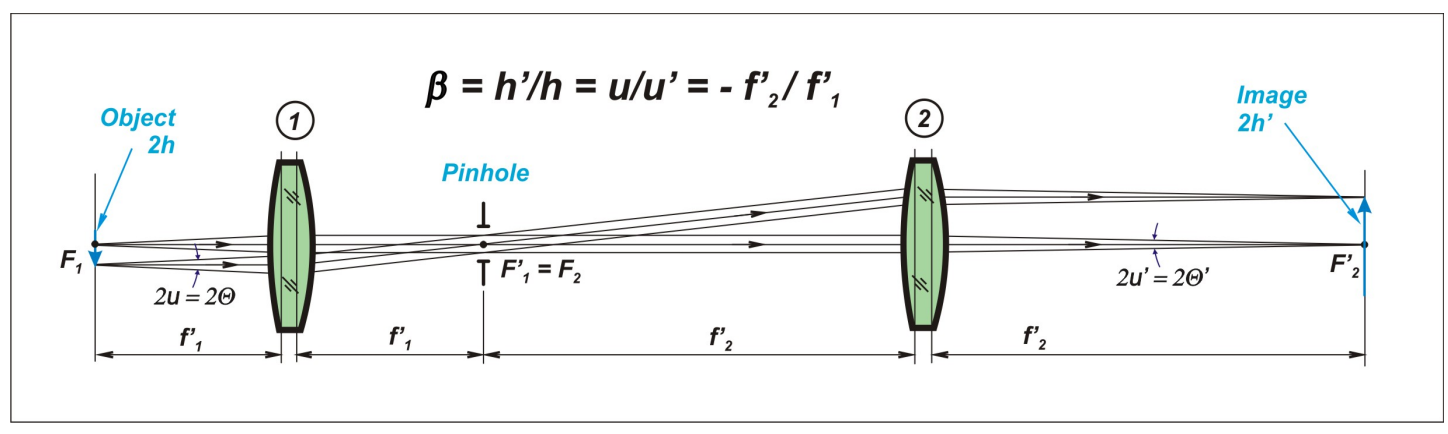

Figure 4 Telecentric imaging.

The optical system providing telecentricity in both spaces of the Object and the Image is composed from two positive optical components in such a way the back focus of the $1^{\text {st }}$ component coincides with the front focus of the $2^{\text {nd }}$ component, i.e. the optical system presents the Keplerian telescope, which famous feature is capability to create real image. Since the optical power of this telecentric system is zero:

- the flat phase front in the Object space is mapped to the flat phase front in the Image space,

- the transverse magnification of the optical system is constant and doesn't depend on position of the Object,

- if the Object is located in front focal plane of $1^{\text {st }}$ component its Image is in back focal plane of $2^{\text {nd }}$ component.

From the point of view of geometrical optics an Image is always created by a beamlet of rays emerging from a particular point of an Object, therefore in Fig. 4 there are shown beamlets of divergence $2 u$ from couple of Object points. In case of laser beams the divergence of beamlets corresponds approximately to divergence of a laser beam $2 \Theta$, i.e. is very small for $\mathrm{TEM}_{00}$ beams, and the irradiance profile behaviour in a telecentric system should be carefully analysed using diffraction theory.

\subsection{Beam shaping layout for Computer Generated Holography}

Let's consider transformation of irradiance profile on example of the optical system to illuminate an SLM, Fig. 5, which was applied at the University of Sheffield ${ }^{11}$ to illuminate the SLM in CGH installation.

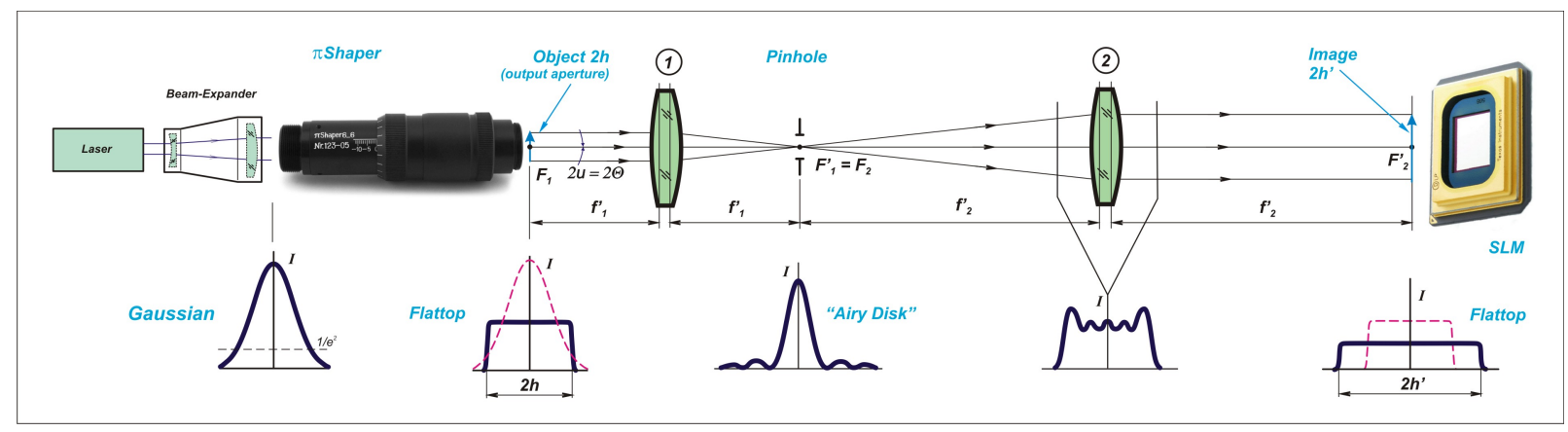

Figure 5 Layout to illuminate a Spatial Light Modulator. 
Divergent Gaussian laser beam from $\mathrm{TEM}_{00}$ fiber coupled laser is transformed by the $\pi$ Shaper to collimated flattop beam, the output of the $\pi$ Shaper is considered as an Object plane for the telecentric imaging system. Since the $\pi$ Shaper conserves low divergence of laser beam, the irradiance profile after it gets transformation due to diffraction that is similar to one shown in Fig. 3. As result near the lenses, Fig. 5, the irradiance distribution isn't uniform, it is typically characterized by appearing some diffraction rings, a particular profile depends on wavelength, beam size and distance from the Object to lenses. According to the diffraction theory the irradiance distribution in a certain plane is result of interference of light diffracted from previous plane of observation. One of well-known conclusions of that theory is similarity of irradiance distribution in optically conjugated Object and Image planes ${ }^{2,3}$ : if the irradiance distribution is uniform in the Object plane, it is uniform in the Image plane as well; and the profile at the $\pi$ Shaper output aperture will be repeated in the Image plane of that aperture, herewith the resulting spot size is defined by transverse magnification $\beta$. Hence, if an SLM is located in the Image plane the incident radiation will be characterized by flat phase front and flat-top intensity profile.

The lenses shown in the example in Fig. 5 are just singlets, but for high quality imaging more sophisticated optical systems should be applied, for example aplanats or microobjectives. Calculation of parameters of a particular imaging setup can be done using well-known formulas of geometrical optics ${ }^{4}$.

A positive lens has a well-known ability to perform two-dimensional Fourier transform ${ }^{2,3}$ and create in its back focal plane irradiance distribution proportional to one in far field. This means in the considered case that irradiance distribution in back focal plane of $1{ }^{\text {st }}$ lens, marked in Fig. 5 as " $F_{1}^{\prime}=F_{2}$ “, is just "Airy disk" described by Eq. (1).

Summarizing results of this example one can see that uniform irradiance after $\pi$ Shaper, the Object plane, is transformed to non-uniform irradiance in area around the lenses, to essentially non-uniform "Airy disk" distribution in back focal plane of $1^{\text {st }}$ lens, and finally is restored to uniform irradiance profile in the Image plane as result of interference of diffracted beam. An important conclusion for practice is that it doesn't matter how the irradiance profile is transformed along the beam path, since the irradiance distribution in the Image plane repeats the Object plane distribution with taking into account transverse magnification. Since the Image is a result of interference of light beams being emitted by the Object and diffracted according to physics of light propagation, it is necessary to take care for transmitting of full light energy through a system and avoid any beam clipping.

Example of applying telecentric imaging optical system at the University of Sheffield ${ }^{10,11}$ to illuminate the SLM in installation of CGH is shown in Fig. 6. A $\pi$ Shaper 6_6_VIS is used to reshape the beam from a laser diode so that a spatial light modulator (SLM), in the form of a Texas Instruments DMD, can be illuminated with a highly coherent, uniform-intensity beam. The DMD is used to project the images of computer-generated holograms that are used for research into photolithography on grossly non-planar substrates ${ }^{13}$. The same system can also be used to illuminate a

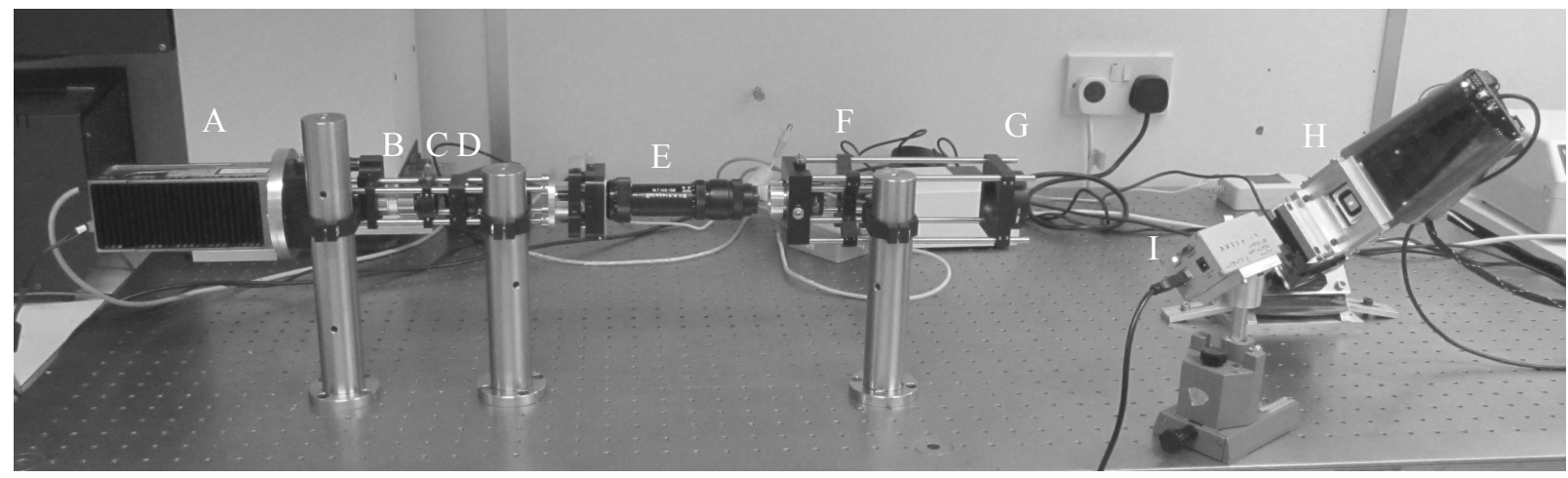

Figure 6 Experimental CGH arrangement for illumination of DMD

A - Diode laser module $\left(\mathrm{P}_{\max }=110 \mathrm{~mW}, \lambda=405 \mathrm{~nm}\right), \mathrm{B}$ - microscope objective, $\mathrm{C}$ - pinhole, $\mathrm{D}$ - collimating lens, $\mathrm{E}-\pi \mathrm{Sh}$ aper $6 \_6$, F- lens, G-collimator, H-DMD (Texas Instruments 0.7" XGA 'DLP' chip, array size $=14 \times 10.5 \mathrm{~mm}$ ), note that the DMD is tilted over at a $45^{\circ}$ angle so that the zero order image reflected from 'on' pixels propagates in the horizontal plane, I - image sensor. 
liquid crystal on silicon (LCoS) SLM. It is essential for these applications to maintain a controlled phase profile across the beam as well as achieving a uniform intensity profile. Initial laser source is the laser diode $\left(1 / \mathrm{e}^{2}\right.$ diameter $\left.=1.6 \mathrm{~mm}\right)$. This beam is spatially filtered using a microscope objective lens and a $25 \mu \mathrm{m}$ diameter pinhole. The spatial filtering can alternatively be accomplished by coupling the laser output into a single-mode optical fiber. The beam is then recollimated using an achromatic doublet lens to produce a clean $5.9 \mathrm{~mm}$ diameter Gaussian beam (Fig. 5) for input into the $\pi$ Shaper. The $\pi$ Shaper 6_6_VIS has the achromatic optical design and is capable of working in the spectral band spanning $405 \mathrm{~nm}$ to $680 \mathrm{~nm}$. The output from the $\pi$ Shaper 6_6_VIS is then further expanded by a 3.5x beam-expander to produce a $21 \mathrm{~mm}$ diameter flat top beam that is sufficiently large to illuminate the entire DMD (Fig. 6). A rectangular aperture close to the DMD can be used to avoid illumination of areas surrounding the micromirror array.

The optical system is used to project CGH images onto non-planar surfaces. Experimental results ${ }^{13}$ demonstrated serious improvement of this technique by homogenizing of illumination of SLM, for example it has become possible to reproduce arbitrary locating in space light objects of arbitrary shape and to realize the 3D-lithography.

\subsection{Optical layout for Holographic Data Storage}

The HDS systems are capable to store extremely large volumes of information; today there are several groups ${ }^{14,15}$ developing modern affordable devices. The optical systems to record and read out the data should provide some specific conditions $^{14}$, and uniformity of intensity distribution with simultaneous flatness of phase front is one of key features of any HDS device. One of implementations of optical system for HDS is shown in Fig. 7.

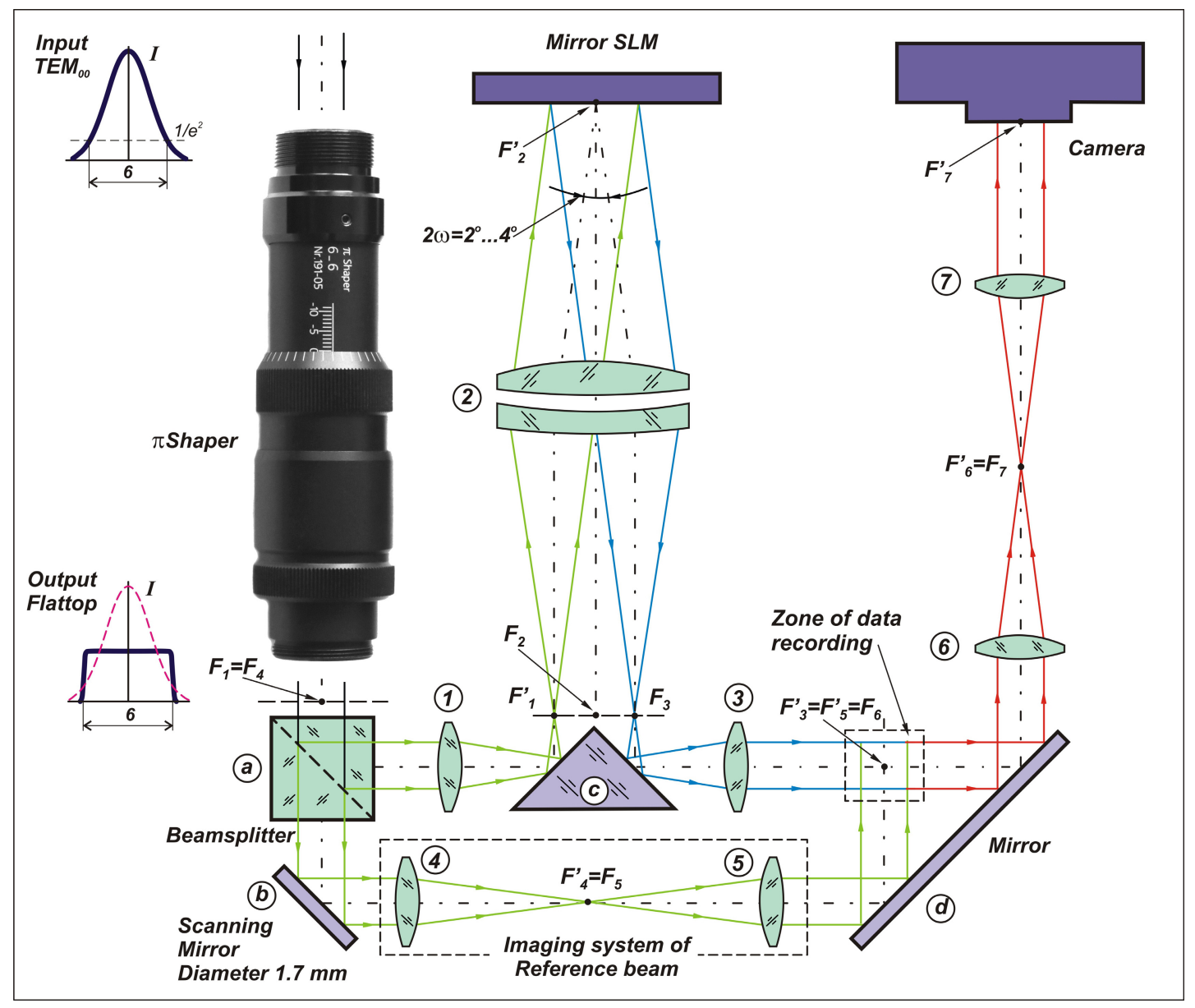

Figure 7. Layout to illuminate a Spatial Light Modulator. 
The basic principle of HDS operation ${ }^{14}$ implies intersection of an Object beam and Reference beam in space where a recording media is located; it is marked in Fig. 7 as "Zone of data recording". Both beams are originated from the same laser beam, which intensity distribution is transformed by a $\pi$ Shaper to uniform one. The collimated flat-top beam is split up into two beams by a cubic Beamsplitter (a). The Reference beam passes to the "Zone of data recording" through mirrors (b) and (d) and telecentric imaging system composed from lenses (4) and (5); the $\pi$ Shaper output coincides with front focal plane (focus $F_{4}$ ) of the lens (4), hence the image is created in back focal plane (focus $F_{5}{ }_{5}$ ) of the lens (5), thus uniform intensity and flat phase front of the Reference beam are provided in the "Zone of data recording".

The Object beam passes to the mirror SLM through telecentric imaging system composed from lenses (1) and (2), the mirror prism (c) is used to bend the optical path; the focuses $F_{1}$ and $F_{2}^{\prime}$ are optically conjugated, hence the SLM is illuminated by a beam of uniform intensity and flat phase front. The field of data signals is created on an SLM, which is implemented as a DMD or mirror LCoS, and is then projected into the "Zone of data recording" through telecentric optical system composed from lenses (2), (3) and mirror prism (c) and realizing the same imaging approach like one of previously described system. As result the Object beam from the SLM interferes with the Reference beam in the "Zone of data recording" and the appearing holographic image is recorded by a recording media, for example lithium niobate $\left(\mathrm{LiNbO}_{3}\right)$ crystal. By tilting the Scanning mirror (b) and changing the field of data signals on SLM one can record large volume of information in a crystal cube. Reading out of the data is realized through illuminating the media with recorded data by the Reference beam and capturing by a Camera the image created by telecentric system composed from lenses (6) and (7).

Thus both tasks of data recording and reading out are solved using telecentric imaging optics with conserving uniformity of laser beam intensity provided by the refractive beam shaper $\pi$ Shaper.

\section{CONCLUSION}

Applying of refractive beam shapers $\pi$ Shaper in SLM-based holography applications makes it possible to provide two basic conditions of the SLM illumination with a laser beam: flat-top irradiance profile and flat phase front, which are mandatory ones for Computer-Generated Holography, Holographic Data Storage, Dot-Matrix hologram mastering, multi-colour Denisyuk holography; these applications get essential benefits from homogenized laser beams: high contrast and equal brightness of reproduced images, higher process reliability and efficiency of laser energy usage, easier mathematical modelling. Availability for various wavelengths, achromatic design, implementations as telescopes and collimators, low divergence and extended DOF make the $\pi$ Shaper unique tools in building SLM-based holography systems. Telecentric imaging systems expand capabilities of $\pi$ Shaper and allow creating image fields of practically unlimited size.

\section{REFERENCES}

[1] Dickey, F.M., Holswade, S.C., [Laser Beam Shaping:Theory and Techniques], Marcel Dekker, New York, (2000).

[2] Goodman, J.W. [Introduction to Fourier Optics], McGraw-Hill, New York, (1996).

[3] Born, M., Wolf E. [Principles of Optics], Cambridge University Press, Cambridge, (1999).

[4] Smith, W.J. [Modern Optical Engineering], McGraw-Hill, New York, (2000).

[5] Hoffnagle, J. A., Jefferson, C. M., "Design and performance of a refractive optical system that converts a Gaussian to a flattop beam", Appl. Opt., vol. 39, 5488-5499 (2000).

[6] Shealy, D.L., Hoffnagle, J.A., "Aspheric Optics for Laser Beam Shaping”, [Encyclopedia of Optical Engineering], Taylor \& Francis (2006).

[7] Kreuzer, J. L. "Coherent light optical system yielding an output beam of desired intensity distribution at a desired equiphase surface." US Patent 3476463, (1969). 
[8] Laskin, A. “Achromatic refractive beam shaping optics for broad spectrum laser applications" Proc. SPIE 7430, Paper 7430-2 (2009).

[9] Laskin, A., “Achromatic Optical System for Beam Shaping” US Patent 8023206, (2011).

[10]Laskin, A., Laskin, V. "Variable beam shaping with using the same field mapping refractive beam shaper" Proc. SPIE 8236, Paper 82360D (2012).

[11]Laskin, A., Williams, G., McWilliam, R., Laskin, V. "Applying field mapping refractive beam shapers to improve holographic techniques" Proc. SPIE 8281, Paper 82810K (2012).

[12] Laskin, A., Laskin, V. "Imaging techniques with refractive beam shaping optics" Proc. SPIE 8490, Paper 8490-19 (2012).

[13] Maiden, A., McWilliam, R. P., Purvis, A., Johnson, S., Williams, G. L., Seed, N. L., Ivey, P. A., "Non-planar photolithography with computer generated holograms", Optics Letters, 30(11), 1300-1302 (2005).

[14] Chao, T.H., Zhou, H.Y., Reyes, G. “Compact Holographic Data Storage System” Proc. IEEE MSS'01 (2001).

[15] Anderson, K., Ayres, M., Sissom, B., Askham, F. "Holographic data storage: rebirthing a commercialization effort" Proc. SPIE 9006, Paper 90060C (2014).

\section{ACKNOWLEDGEMENTS}

The authors are thankful to $\pi$ Shaper users in Sheffield University for their active and patient work with $\pi$ Shaper and kind permission to publish some results of experiments. 